\section{Melanom: kombinierte Checkpointblockade}

\author{
Der Immuncheckpointblocker Ipilimumab hat die Behandlung des \\ fortgeschrittenen malignen Melanoms verbessert. Eine kombinierte \\ Inhibition verschiedener Checkpointsignalwege könnte noch mehr bringen.
}

pilimumab wurde als erster antiCTLA4-Antikörper zur Melanomtherapie zugelassen. Etwa $11 \%$ der Patienten sprechen objektiv auf diesen Verstärker der zytotoxischen T-Zellfunktionen an - ein Rate, die sich möglicherweise durch Kombination mit weiteren Checkpointinhibitoren erhöht. Ein Kandidat ist Nivolumab, ein gegen den PD1 („programmed death 1“)-Rezeptor gerichteter monoklonaler Antikörper, der bei Patienten mit fortgeschrittenem Melanom ebenfalls wirksam ist. Die Blockade von CTLA4 und PD1 zeigte in einer Phase-I-Studie hohe Ansprechraten.

In einer Phase-III-Studie wurde bei 142 therapienaiven Patienten mit metasta- siertem Melanom Nivolumab plus Ipilimumab mit der Ipilimumab-Monotherapie anhand des primären Studienparameters objektives Ansprechen verglichen. Die minimale Nachbeobachtungszeit betrug dabei elf Monate.

Unter der Kombination zeigten 44 von 72 Patienten mit BRAF-V600-WildtypTumor ein objektives Ansprechen im Vergleich zu vier von 37 Patienten unter Ipilimumab-Monotherapie (61\% vs. $11 \%$; $\mathrm{p}<0,001)$. Komplett sprachen 16 Patienten (22\%) in der Kombinations- und kein einziger in der Kontrollgruppe an. Die mediane Dauer des Ansprechens war zum Zeitpunkt der Publikation in keiner Gruppe erreicht, das mediane progressi- onsfreie Überleben mit 4,4 Monaten nur in der Ipilimumab-Monotherapiegruppe (Hazard Ratio 0,40; $\mathrm{p}<0,001$ ). Vergleichbare Ergebnisse für Ansprechen und progressionsfreies Überleben ließen sich auch bei 33 Patienten mit BRAF-mutierten Tumoren beobachten. Medikationsassoziierte Nebenwirkungen vom Grad $3 / 4$ traten bei $54 \%$ der Patienten unter der Kombinations- und bei $24 \%$ unter der Monotherapie auf.

Fazit: Die kombinierte Inhibition durch Nivolumab plus Ipilimumab verbessert bei Patienten mit fortgeschrittenem, therapienaivem Melanom deutlich objektives Ansprechen und progressionsfreies Überleben gegenüber der IpilimumabMonotherapie. Nebenwirkungen sind unter der Kombination vermehrt, aber akzeptabel. Dr. Barbara Kreutzkamp

Postow MA et al. Nivolumab and ipilimumab versus ipilimumab in untreated melanoma. $\mathrm{N}$ Engl J Med 2015; 372: 2006-17

Nekrotisierte Melanommetastase

\title{
Durchschlagende Immuntherapie
}

Berichtet wird der Fall einer 49-jährigen Patientin, die sich im New Yorker Memorial Sloan Kettering Cancer Center mit einer mehr als faustgroßen gestielten nekrotischen Masse unterhalb der linken Brust vorstellte (Abb. links). Vier Jahre zuvor war bei der Patientin am Rücken ein Melanom mit 4,2 mm Durchmesser chirurgisch entfernt worden. Die Frau hatte daraufhin eine einjährige Therapie mit Interferon a erhalten. Ein aktuell angefertigtes CT-Scan zeigte eine Lymphadenopathie in der linken Mamma. Ein Jahr zuvor war bereits eine subkutane Neubildung reseziert worden, die sich als BRAF-Mutation-positives Melanom herausgestellt hatte.

Das Ärzteteam leitete unter der Annahme, dass es sich bei dem Tumor unter der Brust um eine Melanommetastase handelte, eine Therapie mit zwei monoklonalen Antikörpern ein: Ipilimumab und Nivolumab ( $3 \mathrm{bzw} .1 \mathrm{mg} / \mathrm{kg}$ ). Beide Substanzen sind zur Behandlung des metastasierten Melanoms zugelassen. Drei Wochen später präsentierte sich die Patientin wieder in der Klinik - mit einem überraschenden Befund: Der Tumor war nach nur einem The- rapiezyklus verschwunden. An seiner Stelle klaffte ein etwa zwei Finger breites Loch (Abb. rechts). Aus der Tumorhöhle entnommenes Gewebe war zytologisch negativ. Ein CT-Scan nach weiteren drei Wochen bestätigte die makroskopische Tumorfreiheit und zeigte, dass auch die Lymphadenopathie zurückgegangen war. Die Antikörpertherapie, die man wegen eines Ausschlags zwischenzeitlich unterbrochen hatte, wurde nun wieder aufgenommen.

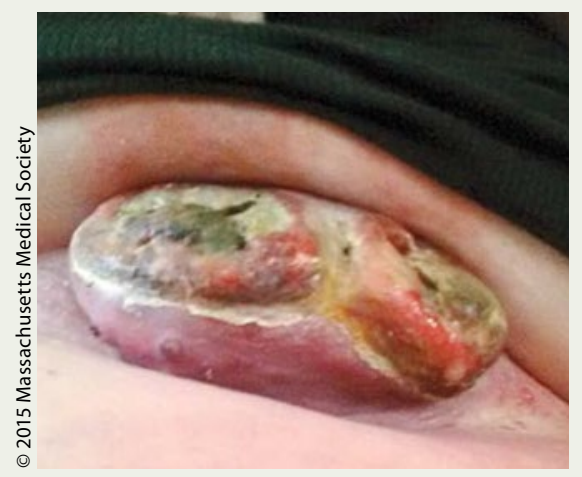

Nekrotisierte Melanommetastase vor (links) und nach (rechts) der Behandlung

Die Autoren nehmen den Fall zum Anlass für eine ungewöhnliche Warnung: Das unter Umständen überaus heftige Ansprechen auf die Immuntherapie könne an anderen Körperstellen gravierende Konsequenzen haben, so etwa bei Tumoren der Darmwand oder des Myokards. Bei der Therapieplanung sei dies zu berücksichtigen. Dr. Elke Oberhofer

Chapman PB et al. Rapid Eradication of a Bulky Melanoma Mass with One Dose of Immunotherapy. N Engl J Med 2015; 372: 2073-4

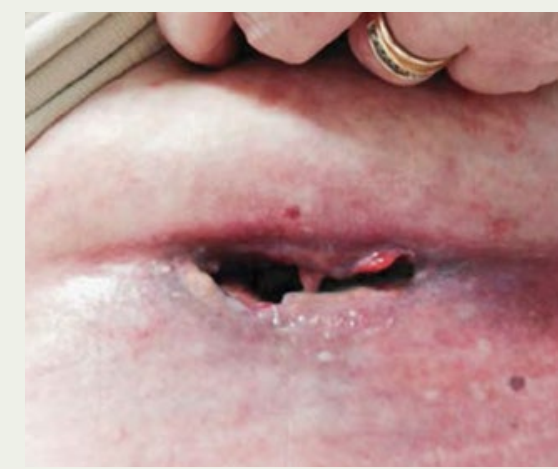

